\title{
The ABCC6 transporter: what lessons can be learnt from other ATP-binding cassette transporters?
}

\section{Olivier M. Vanakker*, Mohammad J. Hosen and Anne De Paepe}

Center for Medical Genetics, Ghent University Hospital, Ghent, Belgium

\section{Edited by:}

Theodore Perkins, Ottawa Hospital

Research Institute, Canada

\section{Reviewed by:}

Scott H. Harrison, North Carolina A\&T State University, USA

Ayse Meric Ovacik, University at Buffalo, USA

\section{${ }^{*}$ Correspondence:}

Olivier M. Vanakker, Center for Medical Genetics, Ghent University Hospital, De Pintelaan 185, B-9000 Ghent, Belgium

e-mail:olivier.vanakker@ugent.be

\begin{abstract}
ABC transporters represent a large family of ATP-driven transmembrane transporters involved in uni- or bidirectional transfer of a large variety of substrates. Divided in seven families, they represent 48 transporter proteins, several of which have been associated with human disease. Among the latter is ABCC6, a unidirectional exporter protein primarily expressed in liver and kidney. ABCC6 deficiency has been shown to cause the ectopic mineralization disorder pseudoxanthoma elasticum (PXE), characterized by calcification and fragmentation of elastic fibers, resulting in oculocutaneous and cardiovascular symptoms. Unique in the group of connective tissue disorders, the pathophysiological relation between the ABCC6 transporter and ectopic mineralization in PXE remains enigmatic, not in the least because of lack of knowledge on the substrate(s) of ABCC6 and its unusual expression pattern. Because many features, including structure and transport mechanism, are shared by many $\mathrm{ABC}$ transporters, it is worthwhile to evaluate if and to what extent the knowledge on the physiology and pathophysiology of these other transporters may provide useful clues toward understanding the (patho)physiological role of ABCC6 and how its deficiency may be dealt with.
\end{abstract}

Keywords: pseudoxanthoma elasticum, $A B C C 6, A B C$ transporters, substrate identification, clinical variability, integrated approach, systems biology, modifier genes
In this paper, we will summarize relevant knowledge and methods for analysis on $\mathrm{ABC}$ transporters which may be useful for the further study of ABCC6.

Pseudoxanthoma elasticum is an autosomal recessive disorder resulting from aberrant mineralization and fragmentation of elastic fibers in the extracellular matrix of the skin, the eyes and the cardiovascular system. It is characterized by papular skin lesions, a retinopathy prone to hemorrhage due to subretinal neovascularisation, and premature occlusive vessel disease, with considerable inter- and intra-familial variability of severity (Vanakker et al., 2008). Thirteen years after the discovery of the causal relation between ABCC6 mutations and PXE, significant progress has been made in the characterization of the $A B C C 6$ gene and the transporter it encodes (Bergen et al., 2000; Le Saux et al., 2000). Nevertheless, ABCC6 remains surrounded by a number of unsolved enigmas, including its substrates and physiological functions as well as the mechanisms by which it is connected to ectopic elastic fiber mineralization. Further, the significant variability in clinical severity of the PXE phenotype cannot be explained by the ABCC6 mutations, as demonstrated by numerous negative genotype-phenotype correlation studies (Meloni et al., 2001; Gheduzzi etal., 2004; Chassaing etal., 2005; Pfendner et al., 2007). This suggested the involvement of modifier genes, influencing particularly the severity of PXE symptoms (Zarbock et al., 2009).

Despite marked structural resemblance with other ABC transporters, ABCC6 has been considered the odd man out, due to its unique relation with a connective tissue disease affecting tissues in which it is hardly expressed. Drawing analogies between $\mathrm{ABCC} 6$ and the other $\mathrm{ABC}$ transporters has been done in the past, particularly to gain insights in substrate interactions. While, after more than a decade, the identification of this substrate is being further pursued, it may be a good time to evaluate if information on other $\mathrm{ABC}$ transporters as well as the difficulties that were and are still encountered in their study may point out potential pitfalls, concepts and approaches on how to deal with the challenges in the field of PXE.

\section{THE PHYSIOLOGICAL FUNCTIONS OF ABCC6}

Perhaps one of the most intriguing questions surrounding ABCC6 is the nature of its substrate and hence the physiological function of the transporter. Following unsuccessful comparisons, it is doubtful that the function of other members of the ABC transporter family will imply anything concerning the actual function of ABCC6 (Madon et al., 2000). It has been previously demonstrated in plant $\mathrm{ABC}$ transporters that - despite phylogenetic similarities and domain homologies - related $\mathrm{ABC}$ transporters can in fact serve diverse physiological functions. In humans, this was endorsed through the comparison of the cystic fibrosis transmembrane conductance regulator (ABCC7 or CFTR, the gene for cystic fibrosis) and P-glycoprotein (ABCB1 or MDR - multidrug resistance protein; Luckie et al., 2003). This however does not imply that nothing can be learned from previous experiences with functional dissection of $\mathrm{ABC}$ transporters. Awareness has risen for several of these that the complexity of (patho)physiological mechanisms related to the wild type and mutant transporter is much higher than anticipated; in this respect and based on our current knowledge, ABCC6 will probably not be an exception to the rule. This enlarging complexity began with the changing concept of 
substrate specificity. For several $\mathrm{ABC}$ transporters either one or a small number of closely related substrates are known. However, there have been reports of other transporters, such as ABCC1, $\mathrm{ABCC} 2$ or $\mathrm{ABCB} 1$, for which multispecificity for a diverse range of substrates has been shown (Jemnitz et al., 2010; He et al., 2011). The diversity of (patho) physiological observations in PXE - oxidative stress, the PXE serum factor, vitamin $\mathrm{K}$ deficiency, the relation with ENPP1, does not make it unthinkable that they result from aberrant transport of more then one substrate, thus influencing more than one physiological process (Le Saux et al., 2006; Pasquali-Ronchetti et al., 2006; Vanakker et al., 2010; Nitschke et al., 2011). For many ABC transporters - including ABCC6 - the specific sequences responsible for substrate recognition have not yet been identified, which may add to the complexity of substrate identification (Glavinas et al., 2004). Nevertheless, for some transporters such as ABCC2 and Pgp, binding-site models have been proposed usingrelatively high accuracy in silico methods. Such models include the presence of one large binding site and multiple smaller ones, primary and secondary binding sites or three distinct sites (Hirono et al., 2005; Borst et al., 2006; Pedersen et al., 2008; Ferreira et al., 2013).

These in silico approaches such as molecular docking, though insufficiently reliable to pinpoint with certainty the physiological substrates, may also have the advantage to generate a list of potential substrates in a cost- and time-efficient manner. Subsequently, these predictions need to be validated in vivo. Several approaches can be applied to identify physiological compounds transported by $\mathrm{ABC}$ proteins, such as in vivo hepatobiliairy elimination studies in mutant animal models, and using membrane vesicles or functional assays based on mass spectrometry (Ci et al., 2007; Katona et al., 2009; Jemnitz et al., 2010). Though murine model organisms are commonly used for such experiments and the Abcc6 knockout mouse model largely recapitulates the clinical features of PXE, there have been reports emphasizing the differences in the physiological mechanisms between rodents and humans, which may slow down substrate identification (Ivan et al., 2013). This has been the case for the ABCG2 protein, recently discovered to be an urate transporter (Nakayama et al., 2011). It should remind us to be cautious to extrapolate findings (positive or negative) in other species to the human disease. Remembering the adage that humans remain the most optimal model to search for substrate(s), an interesting approach for substrate screening has been reported for ABCC2 (Krumpochova et al., 2012). To determine the extent of its substrate spectrum, a variant on the classic vesicular transport experiments has been applied to extract substrates from body fluids. Classic vesicular transport studies have as a drawback that an upfront hypothesis about the transported compounds is necessary and that only one substrate can be evaluated at a time. Certainly, the necessity of an upfront hypothesis is a major disadvantage with respect to ABCC6. By incubating the vesicles in body fluids (in the case of $A b c c 2$ murine urine) and analyzing the vesicle content by LC/MS, several novel compounds were identified. To apply this technique for ABCC6 would imply the use of human plasma and/or hepatocytes. This transportomics technique has great potential with several advantages including a reduced number of experimental animals and would mean that identification of compounds is not a prerequisite to study their transport in vesicular transport experiments as one can fish for new substrates. Combining transportomics with untargeted metabolomics analysis would further increasethe range of potential substrates that can be identified. Disadvantages of the technique include that it is less suitable for identifying hydrophobic substrates. The complex composition of body fluids may require. fractionation to limit the effect of regulators of the transporter which could mask transport of some substrates (Krumpochova et al., 2012).

After several years of tranquility, the expression profile of ABCC6 has again been the subject of debate. ABCC6 is predominantly expressed at the basolateral side of liver and kidney cells, though the transporter also has differential expression in the gut and gastro-intestinal tract (Sinkó et al., 2003; Mutch, 2004; Pomozi et al., 2013). Recently, a supposed intracellular location in the mitochondria-associated membrane (MEM) - part of the ER complex - has been described (Martin et al., 2012). Though the shift in the paradigm linking the expression and function of ABCC6 to the hepatic and renal plasma membrane (PM), which is declared in this paper, should be reviewed with skepticism - the amount of evidence locating ABCC6 in the PM is after all overwhelming - the concept of an additional intracellular localization is potentially interesting and may further increase the complexity of the pathophysiological enigma at hand. This issue has been the topic of debate in recent papers by respectively Martin et al. (2012, 2013) and Pomozi et al. (2013). Demonstrating again the PM localization of ABCC6, the latter group could not confirm the MEM localization while the former called on methodological arguments to defend their findings (Martin et al., 2013; Pomozi et al., 2013). Challenging the cellular localization of proteins is not unprecedented in the ABC superfamily. Recently, the position of the ABCB6 transporter, originally thought to function in mitochondrial porphyrin metabolism, was challenged and extensively documented with experimental and literature data (Kiss et al., 2012). Despite the critical review and convincing evidence of the true physiological function of ABCB6, the authors did not completely exclude a contribution of ABCB6 to porphyrin metabolism and appealed for further and thorough study of the true pathophysiological function(s) of this transporter (Kiss et al., 2012). The knowledge on the subcellular localization of a native protein is of critical importance to model and understand the pathophysiology of any disease. Therefore, unity should be achieved regarding the PM localization of the ABCC6 transporter, but as with ABCB6 I would at this point not completely reject the idea of an additional intracellular localization of ABCC6. In view of the current contradictory results on the potential MEM localization, further work should be done to clarify this issue as the abnormal mitochondrial morphology and membrane potential in PXE - mentioned by Martin et al. (2012) as supporting evidence is insufficient; indeed, it has been demonstrated for the renal ABCB1 transporter that morphological abnormalities of the mitochondria can occur in the absence of a mitochondrial localization, possibly due to accumulation of toxic products (Huls et al., 2007). It cannot be excluded that ABCC6 may move between different membrane compartments under particular conditions, which theoretically may explain the contradicting findings for MEM localization. This has been shown for the PM ABCA1 transporter which, in the absence of extracellular Apo-AI, can be sequestered on intracellular membranes or 
degraded (Tang et al., 2009). As one particular protein can serve strictly different functions in separate parts of the same cell - in view of the complexity of what we know on PXE, this might not even be so unexpected - a validated intracellular localization of ABCC6 may help us further to understand the disease (Kumar and Snyder, 2002).

Though much attention has been paid to the expression of the ABCC6 transporter in the liver, its presence in the kidney, gut and the intestinal tract are less frequently the focus of attention. It has been shown for ABC transporters such as ABCC2 that the $\mathrm{ABC}$ protein may have different organ-specific functions (Jemnitz et al., 2010). Where in the liver it functions in biliairy transport, in the kidney it is involved in the excretion of small organic anions. A similar tissue-dependent function was shown for ABCA1 and ABCG1 (Tarling et al., 2013). Specifically addressing these extrahepatic ABCC6 proteins is necessary as it is not unconceivable that for example the mucosal involvement in PXE may depend on or be modulated by the intestinal ABCC6 transporter.

\section{REGULATION OF ABCC6}

Distinct pathways are involved in the regulation of $\mathrm{ABC}$ transporters, comprising genetic, epigenetic and nuclear-receptor mediated mechanisms, as well as post-transcriptional target repression by microRNAs (miRNAs), which can be triggered by hormones, growth factors and exogenous factors. Several examples exist of ABC transporters, such as Pgp, MRP4, BCRP, whose regulation depends on a combination of mechanisms (Masereeuw and Russel, 2012). Nuclear factor regulation and methylation dependent epigenetic regulation has been described for ABCC6 (Arányi et al., 2005; de Boussac et al., 2010; Ratajewski et al., 2012). So far, no correlation with the PXE phenotype, its variability or potential therapeutic approaches has been attempted. The influence of epigenetic changes, such as promotor DNA methylation, on disease variability has been shown for ABCA1 and coronary artery disease and various drug transporters such as ABCB1and MDR1 (Baker et al., 2005; Reed et al., 2008; Guay et al., 2012). Therapeutic consequences may include the use of histone deacytelase inhibitors, as reported for MDR1 (Jiang et al., 2009).

MicroRNA is a family of short non-coding RNAs involved in the negative regulation of gene expression at the posttranscriptional level (He and Hannon, 2004). With a few hundred miRNAs identified, it is estimated that $50 \%$ of the protein-coding genes are regulated by them. Among ABC transporters, miRNA dependent regulation has been documented for CFTR, MRP2 and 4, $P g P$ and $B C R P$, either directly or indirectly through nuclear factors (Borel et al., 2012; Oglesby et al., 2013). In the CFTR gene, variants have been described that may influence the miRNA target sites in the $3^{\prime}$ UTR (Amato et al., 2013). It has been suggested that certain single nucleotide polymorphisms (SNPs) can influence the affinity for inhibitory miRNAs and may explain the differences in clinical expression between patients with an identical genotype. Mutations in the $3^{\prime}$ UTR have also been described in the $A B C C 6$ gene, so the involvement of miRNAs in PXE pathophysiology may not be purely theoretical. Identification of such miRNAs does not only open the possibility of using modulators, such as chemically engineered oligonucleotides called antagomirs, with the goal of influencing mechanisms that underlie disease initiation or progression (Masereeuw and Russel, 2012). Of interest is that miRNAs have been demonstrated useful biomarkers in kidney disease (Amato et al., 2013; De Guire et al., 2013). The development of an accurate biomarker set for PXE, which does not yet exist, will enable clinical studies to determine whether a compound has a clinically significant effect on the PXE phenotype.

\section{CLINICAL VARIABILITY}

The clinical variability of the PXE phenotype remains a challenge for patients and physicians, making an individualized approach at this moment nearly impossible. Because of the lack of correlations between the patients phenotype and ABCC6 genotype, the possibility of modifier genes has been suggested (Hendig et al., 2007; Zarbock et al., 2010, 2009). The number of potential modifier genes in PXE is currently still limited, which is not totally unexpected; for many ABC transporters the identification of clinical modulators remains challenging. The clinical variability in PXE shows similarities with the variability in cystic fibrosis (CF), the clinical course of which is also difficult to predict using the ABCC7 mutations. Consequently, the quest for modifier genes has started and several modifiers of pulmonary outcome in CF have been described (Blaisdell et al., 2004; Boyle, 2007; Weiler and Drumm, 2012; von Kanel et al., 2013). The search for CF modifiers has lead to several recommendations which are equally valid for ABCC6. First, the importance of an in depth, unambiguous and universal definition of the phenotype has been deemed extremely important. Indeed, the description of the clinical features of the PXE phenotype is often inconsistent in different reports, even when tools such as the Phenodex ${ }^{\circledR}$ are available (Pfendner et al., 2007). A more standardized definition of the phenotypic features will allow a more reliable identification and comparison of modifiers. Further, the limitations of association studies where the relationship between phenotype and polymorphisms in candidate modifier genes is examined have become clear, with the possibility of false positive studies or the causal effect of other genes which may travel with the candidate gene(s) (Nadeau, 2001; Accurso and Sontag, 2003). Finally, a study in twins and siblings in CF indicates that functions directly related to CFTR, membrane ion transport and/or intracellular trafficking of mutant protein are subject to modifier effects (Bronsveld et al., 2000, 2001). Identification of modifiers for functions directly related to the ABC transporter may also yield further insights in the pathophysiology of PXE and provide novel therapeutic targets. Next generation sequencing (NGS), a revolutionizing sequencing technique enabling parallel sequencing of multiple genes and whole exome sequencing is also an opportunity to identify modifier genes and variants, particularly in rare disorders in which large cohorts are often difficult to gather. By combining analysis of extreme phenotypes with pathway analysis, significant power can still be obtained, as was demonstrated by using NGS in CF (Emond et al., 2012).

Modifier genes may not be the only mechanism involved in the variability of PXE. For several ABC transporters, compensatory mechanisms have been described. These include upregulation of MRP3 expression in Dubin-Johnson syndrome, thus compensating for the impaired ABCC2 function (Masereeuw and Russel, 2012). Such compensatory mechanisms have also been suggested for PXE. Gene expression profiling of ABC transporters in dermal 
fibroblasts revealed increased expression of seven genes, including $A B C C 2$ and several members of the A-subfamily (Hendig et al., 2008). The latter was further explored in hepatocytes of a knock-out mouse model where tissue specific upregulation of Abca4 was demonstrated in the liver (Li and Uitto, 2011). However, no further studies have investigated other potential compensatory mechanisms, though they may be of significant importance in understanding PXE, for aiming a more personalized follow-up of patients and for the introduction of novel therapeutic approaches.

\section{AN INTEGRATIVE APPROACH FOR PXE RESEARCH}

Pseudoxanthoma elasticum is one of the diseases where, through the dedicated work of a relatively small group of researchers, a large number of data and observations are gathered. To take on the challenges that we are facing in the field of PXE, integration of all these data and findings may ultimately be the most difficult though imperative step to move forward efficiently. One interesting initiative in this respect is the Clinical and Functional Translation of CFTR (CFTR2) project, which presents a novel approach to clinical and functional annotation of mutations in the CFTR gene. Within this project, clinical and molecular data are gathered from CF registries and centers, in a standardized

\section{REFERENCES}

Accurso, F. J., and Sontag, M. K. (2003). Seeking modifier genes in cystic fibrosis. Am. J. Respir. Crit. Care Med. 167, 289-290. doi: $10.1164 / \mathrm{rccm} .2210006$

Amato, F., Seia, M., Giordano, S., Elce, A., Zarrilli, F., Castaldo, G., etal. (2013). Gene mutation in microRNA target sites of CFTR gene: a novel pathogenetic mechanism in cystic fibrosis? PLoS ONE 8:e60448. doi: 10.1371/journal.pone. 0060448

Arányi, T., Ratajewski, M., Bardóczy, V., Pulaski, L., Bors, A., Tordai, A., et al. (2005). Identification of a DNA methylation-dependent activator sequence in the pseudoxanthoma elasticum gene, ABCC6. J. Biol. Chem. 280, 18643-18650. doi: 10.1074/jbc.M501139200

Baker, E. K., Johnstone, R. W., Zalcberg, J. R., and El-Osta, A. (2005). Epigenetic changes to the MDR1 locus in response to chemotherapeutic drugs. Oncogene 24, 8061-8075. doi: 10.1038/sj.onc. 1208955

Bergen, A. A., Plomp, A. S., Schuurman, E. J., Terry, S., Breuning, M., Dauwerse, H., et al. (2000). Mutations in ABCC6 cause pseudoxanthoma elasticum. Nat. Genet. 25, 228-231. doi: 10.1038/76109

Blaisdell, C. J., Howard, T. D., Stern, A., Bamford, P., Bleecker, E. R., and Stine, O. C. (2004). CLC2 single nucleotide polymorphisms (SNPs) as potential modifiers of cystic fibrosis disease severity. BMC Med. Genet. 5:26. doi: 10.1186/14712350-5-26

Borel, F. F., Han, R. R., Visser A. A., Petry, H. H., van Deventer, S. J., Jansen, P. L., et al. (2012). Adenosine triphosphatebinding cassette transporter genes up-regulation in untreated hepatocellular carcinoma is mediated by cellular microRNAs. Hepatology 55, 821-832. doi: 10.1002/hep. 24682

Borst, P. P., Zelcer, N. N., van de Wetering, K. K., and Poolman, B. B. (2006). On the putative cotransport of drugs by multidrug resistance proteins. FEBS Lett. 580, 9-9. doi: 10.1016/j.febslet.2005. 12.039

Boyle, M. P. (2007). Strategies for identifying modifier genes in cystic fibrosis. Proc. Am. Thorac. Soc. 4, 52-57. doi: 10.1513/pats.200605-129JG

Bronsveld, I., Mekus, F., Bijman, J., Ballmann, M., de Jonge, $H$. R., Laabs, U., et al. (2001). Chloride conductance and genetic background modulate the cystic fibrosis phenotype of Delta F508 homozyInvest. 108, 1705-1715. doi: 10.1172/ JCI12108

Bronsveld, I., Mekus, F., Bijman, J. Ballmann, M., Greipel, J., Hundrieser, J., et al. (2000). Residual chloride secretion in intestinal tissue of deltaF508 homozygous twins and siblings with cystic fibrosis. gous twins and siblings. J. Clin.

way and under the control of data managers. Further, data on functional assessment of mutations was added (Castellani and CFTR2 team, 2013). Though valuable initiatives have been taken to establish mutation database for ABCC6, and linking these molecular data to phenotypical characteristics, no such comprehensive database is currently available. A comprehensive database would improve our ability to identify biomarkers and interpret underlying mechanisms of disease variability in PXE. This database would ideally incorporate information on functional mutation data, potential or established modifier variants, exome sequencing data, serum measurements of patients, fibroblast observations, proteomics, metabolomics and other -omics in clinically wellcharacterized patients. This is expected to enhance diagnostics, carrier testing and screening, genotype-phenotype correlations, modifier analysis and insights into pathogenesis and therapies. It can be concluded that the other members of the ABC transporter family can provide us with valuable information and useful precedents for further characterizing the ABCC6 transporter. Perhaps the most important lesson to incorporate in current PXE research is the concept that only an integrative approach will finally enable us to elucidate this disease completely. To this purpose, it is therefore imperative that joint initiatives can be outlined to merge and integrate past, present and future research data.

The European CF Twin and Sibling Study Consortium. Gastroenterology 119, 32-40. doi: 10.1053/gast.2000. 8524

Castellani, C., and CFTR2 team. (2013). CFTR2: how will it help care? Paediatr. Respir. Rev. 14(Suppl. 1), 2-5. doi: 10.1016/j.prrv.2013.01. 006

Chassaing, N., Martin, L., Calvas, P., Le Bert, M., and Hovnanian, A (2005). Pseudoxanthoma elasticum: a clinical, pathophysiological and genetic update including 11 novel ABCC6 mutations. J. Med. Genet. 42, 881-892. doi: 10.1136/jmg.2004. 030171

Ci, L., Kusuhara, H., Adachi, M., Schuetz, J. D., Takeuchi, K., and Sugiyama, Y. (2007). Involvement of MRP4 (ABCC4) in the luminal efflux of ceftizoxime and cefazolin in the kidney. Mol. Pharmacol. 71, 1591-1597. doi: 10.1124/mol.106. 031823

de Boussac, H., Ratajewski, M. Sachrajda, I., Köblös, G., Tordai, A., Pulaski, L., etal. (2010). The ERK1/2-hepatocyte nuclear factor 4alpha axis regulates human ABCC6 gene expression in hepatocytes. J. Biol. Chem. 285, 2280022808. doi: 10.1074/jbc.M110 105593

De Guire, V., Robitaille, R., Tétreault, N., Guérin, R., Ménard, C., Bambace, N., et al. (2013). Circulating miRNAs as sensitive and specific biomarkers for the diagnosis and monitoring of human diseases: promises and challenges. Clin. Biochem. 46, 846-860. doi: 10.1016/j.clinbiochem.2013.03. 015

Emond, M. J., Louie, T., Emerson, J., Zhao, W., Mathias, R. A., Knowles, M. R., et al. (2012). Exome sequencing of extreme phenotypes identifies DCTN4 as a modifier of chronic Pseudomonas aeruginosa infection in cystic fibrosis. Nat. Genet. 44, 886-889. doi: 10.1038/ ng. 2344

Ferreira, R. J., Ferreira, M. J., and Santos, Dos, D. J. (2013). Molecular docking characterizes substratebinding sites and efflux modulation mechanisms within P-glycoprotein. J. Chem. Inf. Model. 53, 1747-6170. doi: $10.1021 / \mathrm{ci} 400195 \mathrm{v}$

Gheduzzi, D., Guidetti, R., Anzivino, C., Tarugi, P., Di Leo, E., Quaglino, D., etal. (2004). ABCC6 mutations in Italian families affected by pseudoxanthoma elasticum (PXE). Hum. Mutat. 24, 438-439. doi: 10.1002/humu.9284

Glavinas, H., Krajcsi, P., Cserepes, J., and Sarkadi, B. (2004). The role of $\mathrm{ABC}$ transporters in drug resistance, metabolism and toxicity. Curr. Drug Deliv. 1, 27-42. doi: 10.2174/15672010434 80036

Guay, S.-P. S., Brisson, D. D., Munger, J. J., Lamarche, B. B., Gaudet, D. D., and Bouchard, L. L. (2012). ABCA1 gene promoter DNA methylation is associated with HDL particle profile 
and coronary artery disease in familial hypercholesterolemia. Epigenetics 7, 464-472. doi: 10.4161/epi.19633

He, L., and Hannon, G. J. (2004). MicroRNAs: small RNAs with a big role in gene regulation. Nat. Rev. Genet. 5, 522-531. doi: $10.1038 / \operatorname{nrg} 1379$

He, S.-M., Li, R., Kanwar, J. R., and Zhou, S.-F. (2011). Structural and functional properties of human multidrug resistance protein 1 (MRP1/ABCC1). Curr. Med. Chem. 18, 439-481. doi: 10.2174/092986711794839197

Hendig, D., Arndt, M., Szliska, C., Kleesiek, K., and Götting, C. (2007). SPP1 promoter polymorphisms: identification of the first modifier gene for pseudoxanthoma elasticum. Clin. Chem. 53, 829-836. doi: 10.1373/clinchem.2006.083675

Hendig, D., Langmann, T., Kocken, S., Zarbock, R., Szliska, C., Schmitz, G., et al. (2008). Gene expression profiling of $\mathrm{ABC}$ transporters in dermal fibroblasts of pseudoxanthoma elasticum patients identifies new candidates involved in PXE pathogenesis. Lab. Invest. 88, 1303-1315. doi: 10.1038/labinvest.2008.96

Hirono, S. S., Nakagome, I. I., Imai, R. R., Maeda, K. K., Kusuhara, H. H., and Sugiyama, Y. Y. (2005). Estimation of the three-dimensional pharmacophore of ligands for rat multidrug-resistance-associated protein 2 using ligand-based drug design techniques. Pharm. Res. 22, 260-269. doi: 10.1007/s01869-005-1869-8

Huls, M., Kramers, C., Levtchenko, E. N., Wilmer, M. J., Dijkman, H. B., Kluijtmans, L. A., et al. (2007). P-glycoprotein-deficient mice have proximal tubule dysfunction but are protected against ischemic renal injury. Kidney Int. 72, 1233-1241. doi: 10.1038/sj.ki.5002522

Ivan, S., Breljak, D., Marija, L., and Hrvoje, B. (2013). Are mice, rats, and rabbits good models for physiological, pharmacological and toxicological studies in humans? Period. Biol. $113,7-26$.

Jemnitz, K. K., Heredi-Szabo, K. K., Janossy, J. J., Ioja, E. E., Vereczkey, L. L., and Krajcsi, P. P. (2010). ABCC2/Abcc2: a multispecific transporter with dominant excretory functions. Drug Metab. Rev. 42, 402-436. doi: 10.3109/03602530903491741

Jiang, Z.-P., Xu, P., Wang, G.P., Zhao, X.-L., and Chen, F.P. (2009). Hypothesizing that histone deacetylase inhibitors can be used to reverse multiple drug resistance. Med. Hypotheses 74, 3-3. doi: 10.1016/j.mehy.2009.07.036
Katona, M., Kiss, K., Angyal, V., Kucsma, N., Sarkadi, B., Takáts, Z., etal. (2009). A mass spectrometry based functional assay for the quantitative assessment of $\mathrm{ABC}$ transporter activity. Rapid Commun Mass Spectrom. 23, 3372-3376. doi: 10.1002/rcm.4259

Kiss, K., Brozik, A., Kucsma, N., Toth, A., Gera, M., Berry, L. et al. (2012). Shifting the paradigm: the putative mitochondrial protein ABCB6 resides in the lysosomes of cells and in the plasma membrane of erythrocytes. PLoS ONE 7:e37378. doi: 10.1371/journal.pone. 0037378

Krumpochova, P. P., Sapthu, S. S., Brouwers, J. F. J., de Haas, M. M. de Vos, R. R., Borst, P. P., etal. (2012). Transportomics: screening for substrates of $\mathrm{ABC}$ transporters in body fluids using vesicular transport assays. FASEB J. 26, 738-747. doi: 10.1096/fj.11-195743

Kumar, A. A., and Snyder, M. M. (2002). Protein complexes take the bait. Nature 415, 123-124. doi: $10.1038 / 415123 a$

Le Saux, O., Bunda, S., VanWart, C. M., Douet, V., Got, L., Martin, L., et al. (2006). Serum factors from pseudoxanthoma elasticum patients alter elastic fiber formation in vitro. J. Invest. Dermatol. 126, 1497-1505. doi: 10.1038/sj.jid.5700201

Le Saux, O., Urban, Z., Tschuch, C., Csiszar, K., Bacchelli, B., Quaglino D., et al. (2000). Mutations in a gene encoding an $\mathrm{ABC}$ transporter cause pseudoxanthoma elasticum. Nat. Genet. 25, 223-227. doi: 10.1038/76102

Li, Q., and Uitto, J. (2011). Expression of the Abca-subfamily of genes in Abcc6-/- mice-upregulation of Abca4. Exp. Dermatol. 20, 452-454. doi: 10.1111/j.1600-0625. 2010.01240.x

Luckie, D., Wilterding, J., Krha, M., and Krouse, M. (2003). CFTR and MDR: ABC transporters with homologous structure but divergent function. Curr. Genomics 4, 225-235. doi: 10.2174/1389202033490394

Madon, J., Hagenbuch, B., Landmann, L., Meier, P. J., and Stieger, B. (2000) Transport function and hepatocellular localization of mrp6 in rat liver. Mol. Pharmacol. 57, 634-641.

Martin, L. J., Lau, E., Singh, H., Vergnes, L., Tarling, E. J., Mehrabian, M., et al. (2012). ABCC6 localizes to the mitochondria-associated membrane. Circ. Res. 111, 516-520. doi: 10.1161/CIRCRESAHA.112.276667

Martin, L., Lau, E., Singh, H., Vergnes, L., Tarling, E., Mehrabian, M., etal. (2013). Response to Pomozi et al's research commentary. Circ Res. 112, e152-e153. doi: 10.1161/ CIRCRESAHA.113.301666

Masereeuw, R., and Russel, F. G. M. (2012). Regulatory pathways for ATPbinding cassette transport proteins in kidney proximal tubules. AAPS J. 14 , 883-894. doi: 10.1208/s12248-0129404-z

Meloni, I., Rubegni, P., De Aloe, G., Bruttini, M., Pianigiani, E., Cusano, R., et al. (2001). Pseudoxanthoma elasticum: point mutations in the ABCC6 gene and a large deletion including also ABCC1 and MYH11. Hum. Mutat. 18, 85. doi: 10.1002/humu.1157

Mutch, D. M. (2004). Regional variations in $\mathrm{ABC}$ transporter expression along the mouse intestinal tract. Physiol. Genomics 17, 11-20. doi: 10.1152/physiolgenomics.00150.2003

Nadeau, J. H. (2001). Modifier genes in mice and humans. Nat. Rev. Genet. 2 , 165-174. doi: 10.1038/35056009

Nakayama, A., Matsuo, H., Takada, T., Ichida, K., Nakamura, T., Ikebuchi, Y., etal. (2011). ABCG2 is a High-capacity urate transporter and its genetic impairment increases serum uric acid levels in humans. Nucleosides Nucleotides Nucleic Acids 30, 1091-1097. doi: 10.1080/15257770.2011.633953

Nitschke, Y., Baujat, G., Botschen, U., Wittkampf, T., Moulin, du, M., Stella, J., et al. (2011). Generalized arterial calcification of infancy and pseudoxanthoma eElasticum can be caused by mutations in either ENPP1 or ABCC6. Am. J. Hum. Genet. 90, 25 39. doi: 10.1016/j.ajhg.2011.11.020

Oglesby, I. K., Chotirmall, S. H., McElvaney, N. G., and Greene, C. M. (2013). Regulation of cystic fibrosis transmembrane conductance regulator by microRNA-145, -223, and -494 is altered in $\triangle F 508$ cystic fibrosis airway epithelium. J. Immunol. 190, 3354-3362. doi: 10.4049/jimmunol.1202960

Pasquali-Ronchetti, I., GarciaFernandez, M. I., Boraldi, F., Quaglino, D., Gheduzzi, D., De Vincenzi Paolinelli, C., et al. (2006). Oxidative stress in fibroblasts from patients with pseudoxanthoma elasticum: possible role in the pathogenesis of clinical manifestations. J. Pathol. 208, 54-61. doi: 10.1002/path.1867

Pedersen, J. M., Matsson, P., Bergström, C. A. S., Norinder, U., Hoogstraate, J., and Artursson, P. (2008). Prediction and identification of drug interactions with the human
ATP-binding cassette transporter multidrug-resistance associated protein 2 (MRP2; ABCC2). J. Med. Chem. 51, 3275-3287. doi: 10.1021/ jm7015683

Pfendner, E. G., Vanakker, O. M., Terry, S. F., Vourthis, S., McAndrew, P. E., McClain, M. R., et al. (2007). Mutation detection in the ABCC6 gene and genotype-phenotype analysis in a large international case series affected by pseudoxanthoma elasticum. J. Med. Genet. 44, 621-628. doi: 10.1136/jmg.2007.051094

Pomozi, V., Le Saux, O., Brampton, C., Apana, A., Iliás, A., Szeri, F., et al. (2013). ABCC6 is a basolateral plasma membrane protein. Circ. Res. 112, e148-e151. doi: 10.1161/CIRCRESAHA.111.300194

Ratajewski, M., de Boussac, H., Sachrajda, I., Váradi, A., and Arányi, T. (2012). ABCC6 expression is regulated by CCAAT/enhancer binding protein activating a primatespecific sequence located in the first intron of the gene. J. Invest. Dermatol. 132, 2709-2717. doi: 10.1038/jid.2012.218

Reed, K., Hembruff, S. L., Laberge, M. L., Villeneuve, D. J., Côté, G. B., and Parissenti, A. M. (2008). Hypermethylation of the $\mathrm{ABCB} 1$ downstream gene promoter accompanies ABCB1 gene amplification and increased expression in docetaxel-resistant MCF-7 breast tumor cells. Epigenetics 3, 270-280. doi: 10.4161/epi.3.5.6868

Sinkó, E., Iliás, A., Ujhelly, O., Homolya, L., Scheffer, G. L., Bergen, A. A. B., et al. (2003). Subcellular localization and N-glycosylation of human ABCC6, expressed in MDCKII cells. Biochem. Biophys. Res. Commun. 308, 263-269. doi: 10.1016/S0006291X(03)01349-4

Tang, C. C., Liu, Y. Y., Kessler, P. S. P., Vaughan, A. M. A., and Oram, J. F. J. (2009). The macrophage cholesterol exporter ABCAl functions as an anti-inflammatory receptor. J. Biol. Chem. 284, 3233632343. doi: 10.1074/jbc.M109. 047472

Tarling, E. J., Vallim, T. Q., de A., and Edwards, P. A. (2013). Role of $\mathrm{ABC}$ transporters in lipid transport and human disease. Trends Endocrinol. Metab. 24, 342-350. doi: 10.1016/j.tem.2013. 01.006

Vanakker, O. M., Leroy, B. P., Coucke, P., Bercovitch, L. G., Uitto, J., Viljoen, D., et al. (2008). Novel clinico-molecular insights in pseudoxanthoma elasticum provide an efficient molecular screening 
method and a comprehensive diagnostic flowchart. Hum. Mutat. 29, 205. doi: 10.1002/humu. 9514

Vanakker, O. M., Martin, L., Schurgers, L. J., Quaglino, D., Costrop, L., Vermeer, C., et al. (2010). Low serum vitamin $\mathrm{K}$ in PXE results in defective carboxylation of mineralization inhibitors similar to the GGCX mutations in the PXE-like syndrome. Lab. Invest. 90, 895-905. doi: 10.1038/labinvest. 2010.68

von Kanel, T., Stanke, F., Weber, M., Schaller, A., Racine, J., Kraemer, R., et al. (2013). Clinical and molecular characterization of the potential CF disease modifier syntaxin 1A. Eur. J. Hum. Genet. doi: 10.1038/ejhg.2013.57 [Epub ahead of print].

Weiler, C. A., and Drumm, M. L. (2012). Genetic influences on cystic fibrosis lung disease severity. Front. Pharmacol. 4:40-40. doi: 10.3389/fphar.2013.00040

Zarbock, R., Hendig, D., Szliska, C., Kleesiek, K., and Gotting, C. (2009). Vascular endothelial growth factor gene polymorphisms as prognostic markers for ocular manifestations in pseudoxanthoma elasticum. Hum. Mol. Genet. 18, 3344-3351. doi: 10.1093/hmg/ ddp259

Zarbock, R., Hendig, D., Szliska, C., Kleesiek, K., and Götting, C.
(2010). Analysis of MMP2 promoter polymorphisms in patients with pseudoxanthoma elasticum. Clin. Chim. Acta 411, 1487-1490. doi: 10.1016/j.cca.2010.06.006

Conflict of Interest Statement: The authors declare that the research was conducted in the absence of any commercial or financial relationships that could be construed as a potential conflict of interest.

Received: 12 July 2013; accepted: 23 September 2013; published online: 16 October 2013.

Citation: Vanakker OM, Hosen MJ and De Paepe A (2013) The ABCC6 transporter: what lessons can be learnt from other ATP-binding cassette transporters? Front. Genet. 4:203. doi: 10.3389/fgene. 2013.00203

This article was submitted to Systems Biology, a section of the journal Frontiers in Genetics.

Copyright (c) 2013 Vanakker, Hosen and De Paepe. This is an open-access article distributed under the terms of the Creative Commons Attribution License (CC BY). The use, distribution or reproduction in other forums is permitted, provided the original author(s) or licensor are credited and that the original publication in this journal is cited, in accordance with accepted academic practice. No use, distribution or reproduction is permitted which does not comply with these terms. 